\title{
Top quark FCNCs in extended Higgs sectors
}

\author{
Shankha Banerjee ${ }^{\mathrm{a}}$ (i), Mikael Chala, Michael Spannowsky \\ Physics Department, Institute of Particle Physics Phenomenology, Durham University, Durham DH1 3LE, UK
}

Received: 14 June 2018 / Accepted: 10 August 2018 / Published online: 27 August 2018

(C) The Author(s) 2018

\begin{abstract}
The large number of top quarks produced at the LHC and possible future hadron colliders allows to study rare decays of this particle. In many well motivated models of new physics, for example in non-minimal compositeHiggs models, the existence of scalar singlets can induce new flavor-violating top decays surpassing the Higgs contribution by orders of magnitude. We study the discovery prospects of rare top decays within such models and develop new search strategies to test these interactions in top pairproduced events at the LHC. We demonstrate that scales as large as $10-50 \mathrm{TeV}$ can be probed. Improvements by factors of $\sim 1.5$ and $\sim 3$ can be obtained at $\sqrt{s}=27 \mathrm{TeV}$ and $\sqrt{s}=100 \mathrm{TeV}$ colliders respectively.
\end{abstract}

\section{Contents}

1 Introduction . . . . . . . . . . . . . . 1

2 Effective Lagrangian . . . . . . . . . . . . . . 2

3 Explicit models . . . . . . . . . . . . . . 3

4 LHC prospects for $t \rightarrow S c, S \rightarrow b \bar{b} \ldots . . . .4$

5 LHC prospects for $t \rightarrow S c, S \rightarrow \gamma \gamma \ldots 6$

6 Conclusions ............... . 7

References ............... 8

\section{Introduction}

Processes mediated via flavour changing neutral currents (FCNC) are very rare within the standard model (SM) of particle physics. Any experimental evidence of such processes will thus serve as a clear signal for new physics. These possible rare processes have garnered strong interest in both the theoretical and experimental communities and have triggered numerous studies in search for FCNCs, particularly in the top production [1-7] and decay channels [8-22]. Looking for such rare processes in the top sector is very lucrative because top quarks are copiously produced at high-energy

a e-mail: shankha.banerjee@ durham.ac.uk hadron colliders, and therefore a large number of events can be expected even if the FCNC top decays are very rare. Furthermore, because of its large mass, the top quark is inherently connected to the electroweak symmetry breaking (EWSB) sector where new physics effects are more likely to be present. Numerous experimental searches have been carried out in the top FCNC sector. Some of these searches include $t \rightarrow \gamma c / u$ [12], $t \rightarrow g c / u$ [16], $t \rightarrow Z c / u$ [18,19] and $t \rightarrow h c / u[20,21]$ in single top production or toppair production. The present experimental bounds on these decays are $\mathcal{B}\left(t \rightarrow \gamma_{c}(u)\right)<1.7(0.13) \times 10^{-3}$ [12], $\mathcal{B}(t \rightarrow g c(u))<4.1(0.2) \times 10^{-4}[16], \mathcal{B}(t \rightarrow Z c(u))<$ $2.4(1.7) \times 10^{-4}[18,19]$ and $\mathcal{B}(t \rightarrow h c(u))<2.2(2.4) \times$ $10^{-3}[20,21]$. Similar conclusions can also be seen from the following references $[7,23,24]$.

A priori, however, there might be other particles lurking around the Electroweak (EW) scale, into which the top quark can possibly decay. Out of the different possibilities, scalar singlets, $S$, constitute a prime example at the level of dimension-four interactions. When the singlet mixes with the SM-like Higgs boson, its production is strongly constrained owing to the increasingly precise Higgs signal strength measurements (central values reaching unity along with decreasing uncertainties) in various channels $[25,26]$ and also from measurements of the $W$-boson mass [27-31]. For a relatively small mixing parameter of $\sin \theta$ varying between 0.2 and 0.35 [32,33], one can however, have a wide range of allowed singlet mass.

Such scalar particles are predicted by some of the best motivated models of new physics, including supersymmetric extensions (e.g., the NMSSM [34]) and Composite Higgs Models (CHM) [35-38]. Moreover, we will see that these scalars can induce FCNCs significantly larger than those mediated by the SM-like Higgs boson [39]. The reason for the above is three-fold; (i) The top FCNCs mediated by a new scalar singlet are generally suppressed by one less power of the heavy physics scale, (ii) in principle, the scalar singlet can have a larger decay width into cleaner final states, such 
as $\ell^{+} \ell^{-}, b \bar{b}$ or $\gamma \gamma$ and (iii) in broad classes of models (for example in CHMs), Higgs mediated FCNCs are forbidden in first approximation [8]. Altogether, top FCNCs mediated by new scalars might be well within the reach of the LHC.

Presently, there are no direct limits on $t \rightarrow q S$. However, one can have strong constraints from $D^{0}-\bar{D}^{0}$ oscillations [24,40,41] which always come about as a product of two $S$ Yukawas, $Y_{c t}$ and $Y_{u t}$ (and also $Y_{u c}$ ). In order to circumvent these constraints, one can always fall back upon scenarios where $Y_{u t}$ is negligibly small. We will argue in Sect. 3, that the $u t$ FCNCs can be vanishingly small compared to their $c t$ counterpart in explicit models.

In this work, we scrutinise the reach of the LHC for top FCNCs in top-pair produced events. We consider the standard leptonic decay of one of the tops, while the other is assumed to decay into $S c$, with either $S \rightarrow b \bar{b}$ or $S \rightarrow \gamma \gamma$ (leptonic decays will be analysed in a later work). In principle, the current experimental searches for $t \rightarrow h c$ might be also sensitive to these signals. However, these searches are only optimised for a $125 \mathrm{GeV}$ scalar resonance and not for the whole range of masses, in which $S$ can potentially lie. Moreover, the latest experimental strategies (see e.g. Ref. [42]) rely on trained BDTs which make them hard to recast for arbitrary scalar masses. In light of these issues, we develop new dedicated analyses tailored for each mass point.

The structure of the paper is as follows. In Sect. 2 we outline a model-independent introduction to the signals of interest. We follow this up in Sect. 3, where we discuss concrete realisations, involving both strongly and weakly coupled models of new physics. This allows us to establish wellmotivated benchmark points (BP). We go on to discuss the analysis for the $b \bar{b}$ channel in Sect. 4 and in Sect. 5, we present the corresponding analysis for the $S \rightarrow \gamma \gamma$ mode for the $14 \mathrm{TeV}$ LHC machine. We finally conclude in Sect. 6, where we also provide an outlook for the high energy colliders by commenting on naive estimations of the reach of hadron colliders at $\sqrt{s}=27 \mathrm{TeV}$ and $\sqrt{s}=100 \mathrm{TeV}$.

\section{Effective Lagrangian}

Let us consider a scenario where the SM Higgs sector is extended by a gauge singlet, $S$, having a mass $m_{S}$ in the EW regime. For low energies, we write the relevant Yukawa Lagrangian as follows ${ }^{1}$

$$
\mathcal{L}=-\overline{\mathbf{q}} \mathbf{L}\left(\mathbf{Y}+\mathbf{Y}^{\prime} \frac{|H|^{2}}{f^{2}}+\tilde{\mathbf{Y}} \frac{S}{f}\right) \tilde{H} \mathbf{u}_{\mathbf{R}}+\text { h.c. }
$$

\footnotetext{
${ }^{1}$ We note that in the absence of effective operators, the interaction of $S$ with the fermions, is negligible. Indeed, such interactions only arise at one loop (and only if $S$ is not a pseudo-scalar; otherwise there
}

where $H=\left[\phi^{+},\left(h+\phi^{0}\right) / \sqrt{2}\right]^{t}$, is the SM-like Higgs doublet, $\mathbf{q}_{\mathbf{L}}\left(\mathbf{u}_{\mathbf{R}}\right)$ denotes the left-handed (right-handed) quarks, $\mathbf{Y}, \mathbf{Y}^{\prime}$, and $\tilde{\mathbf{Y}}$ are arbitrary flavour matrices, $v \sim 246 \mathrm{GeV}$, is the Higgs vacuum expectation value (vev) and $f \gtrsim \mathcal{O}(\mathrm{TeV})$ is the new physics scale. In general, the flavour matrices are not aligned, and thus the FCNCs can arise in the EW phase. Among various new physics effects, these induce top flavourviolating decays, viz., $t \rightarrow h c$ or $t \rightarrow S c$. In general, the latter dominates over the former, because (i) $t \rightarrow h c$ is further suppressed by an additional factor of $1 / f$ and (ii) in several UV-complete models, $\mathbf{Y}$ and $\mathbf{Y}^{\prime}$ are approximately aligned. Finally, after the EWSB, one obtains

$$
\begin{aligned}
\mathcal{L} & =-\frac{v}{\sqrt{2}}\left[\overline{\mathbf{q}_{\mathbf{L}}} \mathbf{Y}\left(1+\frac{h}{v}\right) \mathbf{u}_{\mathbf{R}}+\frac{S}{f} \overline{\mathbf{q}_{\mathbf{L}}} \tilde{\mathbf{Y}} \mathbf{u}_{\mathbf{R}}+\mathcal{O}\left(\frac{1}{f^{2}}\right)\right] \\
& \supset \tilde{g} \frac{m_{t}}{f} \overline{t_{L}} S c_{R}+\text { h.c. }
\end{aligned}
$$

where $m_{t} \sim 173 \mathrm{GeV}$ is the top mass and $g$ is an $\mathcal{O}(1)$ coupling. Such interactions can be tested to a high accuracy through rare top decays. Upon using Eq. 2.2, one obtains the partial width of $t \rightarrow S c$ as follows

$\Gamma(t \rightarrow S c)=\frac{\tilde{g}^{2}}{32 \pi} \frac{v^{2}}{f^{2}} m_{t}\left(1-\frac{m_{S}^{2}}{m_{t}^{2}}\right)^{2}$,

and thus for a benchmark point with $\tilde{g} \sim 1$ and $f \sim 1$ $\mathrm{TeV}$, one obtains $\mathcal{B}(t \rightarrow S c) \sim \Gamma(t \rightarrow S c) / \Gamma_{t}^{\mathrm{SM}} \sim 0.03$ with $\Gamma_{t}^{\mathrm{SM}} \sim 1.4 \mathrm{GeV}$ [43]. A full exploration of this decay in singly or pair-produced top quarks at colliders, depends also on how $S$ decays to SM particles. Motivated by CHMs (as discussed below), we consider a scenario where $S$ couples to the SM fermions, $\psi$, via $\frac{c_{\psi} m_{\psi}}{f} S \bar{\psi} \psi$ and to the photons via $\frac{c_{\gamma} \alpha}{4 \pi f} S F_{\mu \nu} \tilde{F}^{\mu \nu}$, where $c_{\psi}$ and $c_{\gamma}$ are arbitrary $\mathcal{O}(1)$ couplings and $\alpha$ is the fine-structure constant. Thus, in the regime, $m_{S} \gg m_{\psi}$, one obtains at leading order

$\Gamma(S \rightarrow \psi \psi)=\frac{N_{c}}{8 \pi} \frac{c_{\psi}^{2} m_{\psi}^{2}}{f^{2}} m_{S} \quad$ and $\quad \Gamma(S \rightarrow \gamma \gamma)=\frac{c_{\gamma}^{2} \alpha^{2}}{64 \pi^{3} f^{2}} m_{S}^{3}$.

Thus, one finds the relation $\mathcal{B}(S \rightarrow \gamma \gamma) / \mathcal{B}(S \rightarrow \bar{\psi} \psi) \sim$ $\frac{\alpha^{2}}{\pi^{2}}\left(m_{S} / m_{\psi}\right)^{2}$. The suppression factor driven by the small electromagnetic coupling can thus be partially compensated upon scaling with the free parameter $m_{S} \cdot \mathcal{B}(S \rightarrow \gamma \gamma)$ can

\section{Footnote 1 continued}

is an accidental symmetry $S \rightarrow-S$, provided $C P$ is conserved). Moreover, these are proportional to the vev of $S$, which triggers the mixing with the Higgs boson and are therefore severely constrained by current Higgs measurements [26,30,31]. In addition, the FCNC currents will be further suppressed by the GIM mechanism. Thus, we would expect $\mathcal{B}(t \rightarrow S c)$ to be several orders of magnitude smaller than $\mathcal{B}(t \rightarrow h c)$, which in the SM is predicted to be smaller than $10^{-13}$ [9]. 
be significantly larger than the $\mathcal{B}(h \rightarrow \gamma \gamma) \sim 2 \times 10^{-3}$, with the precise value being model dependent.

\section{Explicit models}

The Lagrangian in Eq. 2.1 appears naturally in several UVcomplete models, for example in CHMs. In these classes of models, $H$ and $S$ are pseudo Nambu-Goldstone Bosons (pNGBs) arising in a new global symmetry breaking $\mathcal{G} / \mathcal{H}$ at a scale $\sim f$. A prime example is the CHM based on the coset $S O(6) / S O(5)$ [44], which is the smallest one that admits four-dimensional UV completion [45]. The generators of this coset can be chosen as

$$
\begin{array}{ll}
T_{i j}^{m n}=-\frac{i}{\sqrt{2}}\left(\delta_{i}^{m} \delta_{j}^{n}-\delta_{i}^{n} \delta_{j}^{m}\right), & m<n \in[1,5], \\
X_{i j}^{m 6}=-\frac{i}{\sqrt{2}}\left(\delta_{i}^{m} \delta_{j}^{6}-\delta_{i}^{6} \delta_{j}^{m}\right), & m \in[1,5] .
\end{array}
$$

Among these, $X^{16}-X^{46}$ expand the coset space of the Higgs doublet, while the broken generator associated to $S$ is provided by $X^{56}$. The SM fermions do not couple directly to the (fully composite) Higgs. Instead, the latter couples to composite fermionic resonances, which in turn mix with the SM fermions, thus explicitly breaking the global symmetry. The Yukawa Lagrangian depends therefore on the quantum numbers of the aforementioned fermionic resonances. For concreteness, we will assume that these fields transform in the fundamental representation 6 of $S O(6)$. The latter can be decomposed as $\mathbf{6}=1+1+\mathbf{4}$ under the custodial symmetry group $S O(4)$. Let us assume that $u_{R}^{i}$ is embedded in both singlets, whereas the one for $q_{L}^{i}$ is fixed. These are listed as

$U_{R_{1}}^{i}=\left(0,0,0,0, \mathrm{i} u_{R}^{i}, 0\right), \quad U_{R_{2}}^{i}=\left(0,0,0,0,0, u_{R}^{i}\right)$

and $Q_{L}^{i}=\frac{1}{\sqrt{2}}\left(i b_{L}^{i}, b_{L}^{i}, i t_{L}^{i},-t_{L}^{i}, 0,0\right)$.

Using the corresponding Goldstone matrix

$$
\begin{aligned}
\mathrm{U} & =\left[\begin{array}{ccc}
1_{3 \times 3} & & \\
1-h^{2} /\left(f^{2}+\Pi\right) & -h s /\left(f^{2}+\Pi\right) & h / f \\
-h s /\left(f^{2}+\Pi\right) & 1-S^{2} /\left(f^{2}+\Pi\right) & S / f \\
-h / f & -S / f & \Pi / f^{2}
\end{array}\right], \\
\Pi & =f^{2}\left(1-\frac{h^{2}}{f^{2}}-\frac{S^{2}}{f^{2}}\right)^{1 / 2}
\end{aligned}
$$

one obtains the Yukawa Lagrangian

$$
\begin{aligned}
L & =-f y_{i j}^{(1)} \overline{\left(\mathrm{U}^{T} Q_{L}^{i}\right)_{6}}\left(\mathrm{U}^{T} U_{R_{1}}^{j}\right)_{6}-f y_{i j}^{(2)} \overline{\left(\mathrm{U}^{T} Q_{L}^{i}\right)_{6}}\left(\mathrm{U}^{T} U_{R_{2}}^{j}\right)_{6}+\text { h.c. } \\
& =-\frac{1}{\sqrt{2}} \overline{q_{L}^{i}} h t_{R}^{j}\left[y_{i j}^{(2)}\left(1-\frac{h^{2}}{f^{2}}-\frac{S^{2}}{f^{2}}\right)^{1 / 2}+i y_{i j}^{(1)} \frac{S}{f}\right]+\text { h.c. }
\end{aligned}
$$

which, to leading order, reads

$L=-\frac{1}{\sqrt{2}} \overline{q_{L}^{i}} h t_{R}^{j}\left[y_{i j}^{(2)}-y_{i j}^{(2)} \frac{h^{2}}{2 f^{2}}+i y_{i j}^{(1)} \frac{S}{f}+\cdots\right]+$ h.c.

Hence, we obtain $\mathbf{Y}_{i j}=-\mathbf{Y}^{\prime}=y_{i j}^{(2)}$ and $\tilde{\mathbf{Y}}_{i j}=i y_{i j}^{(1)}$. Thus, to leading order, scalar mediated FCNCs are only driven by $S$, provided that $y_{i j}^{(1)}$ and $y_{i j}^{(2)}$ are not aligned. Even in that case, the FCNCs would still arise in the presence of higherdimensional operators, and then undergo suppression by a factor of $1 / g_{*}^{2}$ (with $g_{*}$ being a strong coupling) just as in the minimal CHM $[8,38]$. Similar results hold for other representations, with the exception of those that respect a $S \rightarrow-S$ parity and those for which the shift symmetry of $S$ remains unbroken, examples being $q_{L}^{i}$ in the $\mathbf{6}, t_{R}^{i}$ in the $\mathbf{1 5}$ [46].

We note that although $\tilde{\mathbf{Y}}_{i j}$ is in principle arbitrary, one can easily expect sizeable top-charm couplings and still have small top-up FCNCs. Indeed, despite being not directly measurable, in common viable ansätze, $\mathbf{Y}$ is hierarchical and nearly block-diagonal, with the maximal mixing occurring in the top-charm sector [47,48]. It can therefore be diagonalised as $\mathbf{Y} \rightarrow \mathbf{L}^{\dagger} \mathbf{Y} \mathbf{R}$, with $\mathbf{L}$ and $\mathbf{R}$ being block-diagonal as well. Moreover, in CHMs the aforementioned hierarchy reflects the fact that heavier fermions couple stronger to the composite sector, so not only to $H$ but also to $S$. One can then easily expect a similar block-diagonal structure for $\tilde{\mathbf{Y}}$. As a consequence, $\mathbf{L} \tilde{\mathbf{Y}} \mathbf{R}$ is also block-diagonal with only the topcharm mixing. Hence, we concentrate on the $t \rightarrow S c$ decay channel. However, because we will not use any explicit $c$ tagging in our analyses, our results can easily be translated for the $t \rightarrow S u$ mode.

We define three Benchmark Points (BP), each including $m_{S}=20,50,80,100,120$ and $150 \mathrm{GeV}$, as follows

$\begin{array}{ll}\text { BP 1: } & \tilde{g}=1.0, \quad f=2 \mathrm{TeV} \Longrightarrow \mathcal{B}(t \rightarrow S c) \sim 10^{-3}-10^{-2} ; \\ \text { BP 2: } & \tilde{g}=1.0, \quad f=10 \mathrm{TeV} \Longrightarrow \mathcal{B}(t \rightarrow S c) \sim 10^{-4}-10^{-3} ; \\ \text { BP 3: } & \tilde{g}=0.1, \quad f=2 \mathrm{TeV} \Longrightarrow \mathcal{B}(t \rightarrow S c) \sim 10^{-5}-10^{-4} ;\end{array}$

We note that, although being a priori relatively light, values of $m_{S}<m_{h} / 2 \sim 62.5 \mathrm{GeV}$ are not necessarily excluded by the LHC constraints on the Higgs width [49]. Actually, the latter translates into an upper bound $\Gamma(h \rightarrow S S) \lesssim 10$ $\mathrm{MeV}$. Given that for a quartic coupling $\lambda_{H S} S^{2}|H|^{2}$ one obtains $\Gamma(h \rightarrow S S) \sim \lambda_{H S}^{2} /(32 \pi) \times v^{2} / m_{h}$, we can evade the aforementioned bound provided $\lambda_{H S}<0.05$. Similarly, derivative interactions $\sim h S \partial h \partial S / f^{2}$, unavoidable 
Table 1 Single field extensions of the SM supplemented with $S$ that induce the FCNC of interest at low energy at tree level. The numbers in parenthesis and the subscript denote the $S U(3)_{c}$ and $S U(2)_{L}$ representations and the hypercharge, respectively. From the top left and clockwise, the different diagram legs represent $q_{L}^{i}, t_{R}^{j}, H$ and $S$, respectively

\begin{tabular}{|c|c|c|c|}
\hline Field & Relevant Lagrangian & Diagram & $\tilde{\mathbf{Y}}_{i j} / f^{2}$ \\
\hline$Q=(1,2)_{1 / 6}$ & $\begin{aligned} L_{Q}= & -m_{Q} \bar{Q} Q+\left(\alpha_{i}^{Q} \bar{Q} S q_{L}^{i}\right. \\
& \left.+\tilde{\alpha}_{j}^{Q} \bar{Q} \tilde{H} u_{R}^{j}+\text { h.c. }\right)\end{aligned}$ & & $\frac{\alpha_{i}^{Q} \tilde{\alpha}_{j}^{Q}}{m_{Q}}$ \\
\hline$U=(1,1)_{2 / 3}$ & $\begin{aligned} L_{U}= & -m_{U} \bar{U} U+\left(\alpha_{i}^{U} \bar{U} H q_{L}^{i}\right. \\
& \left.+\tilde{\alpha}_{j}^{U} \bar{U} S u_{R}^{j}+\text { h.c. }\right)\end{aligned}$ & & $\frac{\alpha_{i}^{U} \tilde{\alpha}_{j}^{U}}{m_{U}}$ \\
\hline$\Phi=(1,2)_{1 / 2}$ & $\begin{aligned} L_{\Phi}= & -\frac{1}{2} m_{\Phi}^{2} \Phi^{2}+\left(\alpha_{i j}^{\Phi} \overline{q_{L}^{i}} \tilde{\Phi} u_{R}^{j}\right. \\
& \left.+\kappa S \Phi^{\dagger} H+\text { h.c. }\right)\end{aligned}$ & & $\frac{\alpha_{i j}^{\Phi} \kappa}{m_{\Phi}^{2}}$ \\
\hline
\end{tabular}

in CHMs, contribute to the Higgs width with an effective $\lambda_{H S} \sim 4 m_{h}^{2} / f^{2} \lesssim 0.05$ for a scale, $f \gtrsim 1.2 \mathrm{TeV}$.

If we consider only weakly-coupled extensions of the $\mathrm{SM}+S$, the Lagrangian in Eq. 2.1 can also be induced at tree level by the fields listed in Table 1 . In particular, this means that the NMSSM [34], which extends the SM scalar sector with an additional $S U(2)_{L}$ doublet with $Y=1 / 2$ (required by SUSY itself) as well as with a singlet (in order to avoid the $\mu$-problem [50]), fits naturally into the targets of our analysis.

\section{LHC prospects for $t \rightarrow S c, S \rightarrow b \bar{b}$}

In this section, we focus on the scenario where the scalar singlet decays to a pair of $b$-quarks, yielding a final state comprised of at least four jets, three of them required to be $b$-tagged and exactly one isolated lepton. As described above, we quantify our results in terms of six benchmark masses, viz., $m_{S}=20,50,80,100,120$ and $150 \mathrm{GeV}$. Our ultimate goal in this section is to derive an upper bound on $\mathcal{B}(t \rightarrow S c, S \rightarrow b \bar{b})$ at $95 \%$ Confidence Level (CL).

We have fixed the $b$-tagging efficiency to $70 \%$. The $c(\ell) \rightarrow b$ mistag rate has been taken as $10 \%(1 \%)$. The most dominant real background ensues from semi-leptonic $t \bar{t} b \bar{b}$ production. Besides, the fully leptonic channel from the aforementioned production mode also contributes. The major fake backgrounds that we consider are the semi-leptonic (and leptonic) $t \bar{t}$ merged up to one extra matrix element parton, the $W b \bar{b}$ process merged up to two extra matrix element partons and $Z b \bar{b}$ also merged up to two extra partons with the $Z$-boson decaying leptonically. For the analysis framework, we use MG5_aMC@NLO v2.6.0 [51] for generating the signal and background samples. We employ the MLM merging scheme [52] embedded in this framework, with appropriate parameter choices. We use very loose parton level cuts, viz., $p_{T}(j)>15 \mathrm{GeV}, p_{T}(b)>15 \mathrm{GeV}$ and $p_{T}(\ell)>10 \mathrm{GeV}$, as well as $|\eta(j)|<4,|\eta(b)|<4$ and $|\eta(\ell)|<3$. Moreover, we require the $\Delta R$ separations to be zero for each pair at the generation level. The cross sections of $t \bar{t}, W b \bar{b}, Z b \bar{b}$ and $t \bar{t} b \bar{b}$ are multiplied by $K$-factors of 1.6, 2.3, 1.25 and 1.13, respectively. While the first one can be found in Ref. [53], the other three have been estimated by computing in MG5_aMC@NLO at NLO in QCD. We use the NNPDF 2.3 [54] at leading order. The analyses in this section and in the next are carried out for the $14 \mathrm{TeV}$ LHC.

Furthermore, we shower the samples with Pythia $8[55] .^{2}$ Finally, at the analysis level, we construct the jets employing the anti- $k_{T}$ [56] algorithm with a jet parameter $R=0.4$ in the FastJet [57] framework. All the jets are required to have $p_{T}>30 \mathrm{GeV}$ and to lie within a pseudorapidity range of $|\eta|<2.5$. Leptons must have a $p_{T}>10$ $\mathrm{GeV}$ and $|\eta|<2.5$. For the isolation, we require that the total hadronic activity around the lepton within a cone of $\Delta R=0.2$ is less than $10 \%$ of its transverse momentum. All the aforementioned selected objects are also required to be separated by $\Delta R>0.4$.

After selecting these events, we look for the closest pair (in terms of $\Delta R$ separation) of $b$-tagged jets and reconstruct the top-quark mass $m_{t}^{\Delta R}$ with the additional hardest jet which is not $b$-tagged. We require this variable to be within a window of $50 \mathrm{GeV}$ from $m_{t}$. With the remaining $b$-tagged jet, we construct the transverse mass variable $m_{T}$ and require it to be less than $200 \mathrm{GeV}$. We show the distributions of $m_{t}^{\Delta R}, m_{T}$ and the reconstructed scalar mass, $m_{S}^{\Delta R}$, after the basic cuts (which include the aforementioned $p_{T}$ cuts as well as a requirement for $3 b$-tagged jets, at least an additional light jet and one isolated lepton) for two signal benchmark points and four dominant backgrounds, in Fig. 1. The mass-independent cutflow tables for the six benchmark points and six backgrounds are listed in Tables 2 and 3 respectively. To optimise each signal region, we impose an additional cut, viz., $0.8 m_{S}<m_{S}^{\Delta R}<m_{S}+10$ $\mathrm{GeV}$. In Table 4, we list the final efficiencies for each signal region after this additional cut on top of the aforementioned ones.

Finally, we show our results in Fig. 2. The left plot shows the $95 \%$ upper limit on $B R(t \rightarrow S c, S \rightarrow b \bar{b})$ and the right plot shows the minimum integrated luminosity to test the aforementioned branching ratio to $10^{-4}$ at $95 \% \mathrm{CL}$.

\footnotetext{
${ }^{2}$ We did not find any significant differences when comparing with another setup using MG5_aMC@NLO v2.1.1 with the showering done with Pythia 6.
} 

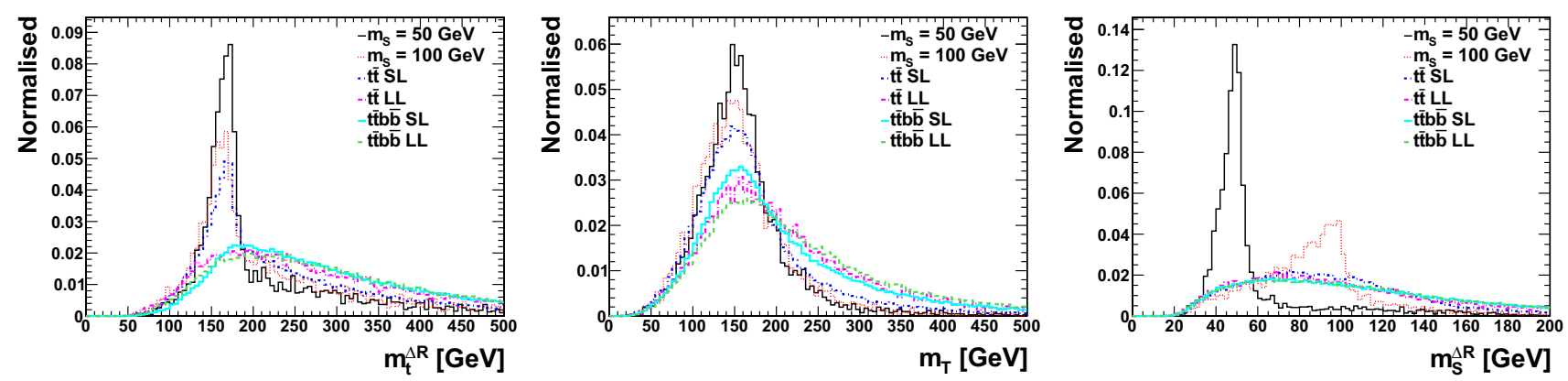

Fig. 1 (Left) The reconstructed top mass from the closest $b$-pair. (Center) The transverse mass $m_{T}$. (Right) The reconstructed mass of the scalar S

Table 2 Efficiency after each cut for the six signal benchmark points

Table 3 Efficiency after each cut for the six dominant backgrounds. SL (LL) denotes semi (di)-leptonic decays

Table 4 Efficiencies $\left(\times 10^{4}\right)$ after the final cut,

$0.8 m_{S}<m_{S}^{\Delta R}<m_{S}+10$ $\mathrm{GeV}$, for each signal benchmark point and for the corresponding backgrounds. SL (LL) denotes semi (di)-leptonic decays

\begin{tabular}{lllllll}
\hline Cuts & $20 \mathrm{GeV}$ & $50 \mathrm{GeV}$ & $80 \mathrm{GeV}$ & $100 \mathrm{GeV}$ & $120 \mathrm{GeV}$ & $150 \mathrm{GeV}$ \\
\hline Basic & 0.014 & 0.050 & 0.051 & 0.056 & 0.063 & 0.063 \\
$\left|\eta_{(b, \ell, j)}\right|<2.5$ & 0.83 & 0.88 & 0.86 & 0.87 & 0.86 & 0.82 \\
$\Delta R($ all pairs $)>0.4$ & 0.96 & 0.94 & 0.93 & 0.93 & 0.94 & 0.94 \\
$\left|m_{t}^{\Delta R}-m_{t}\right|<50 \mathrm{GeV}$ & 0.29 & 0.63 & 0.57 & 0.55 & 0.49 & 0.41 \\
$m_{T}<200 \mathrm{GeV}$ & 0.72 & 0.56 & 0.87 & 0.85 & 0.83 & 0.74 \\
\hline
\end{tabular}

\begin{tabular}{lllllll}
\hline Cuts & $t \bar{t}(\mathrm{SL})$ & $t \bar{t}(\mathrm{LL})$ & $W b \bar{b}$ & $Z b \bar{b}$ & $t \bar{t} b b(\mathrm{SL})$ & $t \bar{t} b b(\mathrm{LL})$ \\
\hline Basic & 0.0038 & 0.0016 & 0.00032 & 0.00016 & 0.11 & 0.073 \\
$\left|\eta_{(b, \ell, j)}\right|<2.5$ & 0.78 & 0.69 & 0.74 & 0.71 & 0.90 & 0.85 \\
$\Delta R($ all pairs $)>0.4$ & 0.95 & 0.94 & 0.95 & 0.95 & 0.96 & 0.91 \\
$\left|m_{t}^{\Delta R}-m_{t}\right|<50 \mathrm{GeV}$ & 0.49 & 0.32 & 0.27 & 0.33 & 0.31 & 0.28 \\
$m_{T}<200 \mathrm{GeV}$ & 0.80 & 0.58 & 0.56 & 0.70 & 0.63 & 0.53 \\
\hline
\end{tabular}

\begin{tabular}{llllllll}
\hline$m_{S}(\mathrm{GeV})$ & Signal & $t \bar{t}(\mathrm{SL})$ & $t \bar{t}(\mathrm{LL})$ & $W b \bar{b}$ & $Z b \bar{b}$ & $t \bar{t} b b(\mathrm{SL})$ & $t \bar{t} b b(\mathrm{LL})$ \\
\hline 20 & 8.2 & 0.12 & 0.037 & 0.017 & 0.0094 & 4.0 & 1.5 \\
50 & 110 & 1.8 & 0.35 & 0.093 & 0.056 & 37 & 17 \\
80 & 140 & 3.4 & 0.60 & 0.080 & 0.070 & 51 & 24 \\
100 & 120 & 3.7 & 0.59 & 0.066 & 0.062 & 49 & 24 \\
120 & 96 & 3.1 & 0.47 & 0.052 & 0.042 & 41 & 19 \\
150 & 51 & 1.4 & 0.23 & 0.025 & 0.019 & 22 & 11 \\
\hline
\end{tabular}
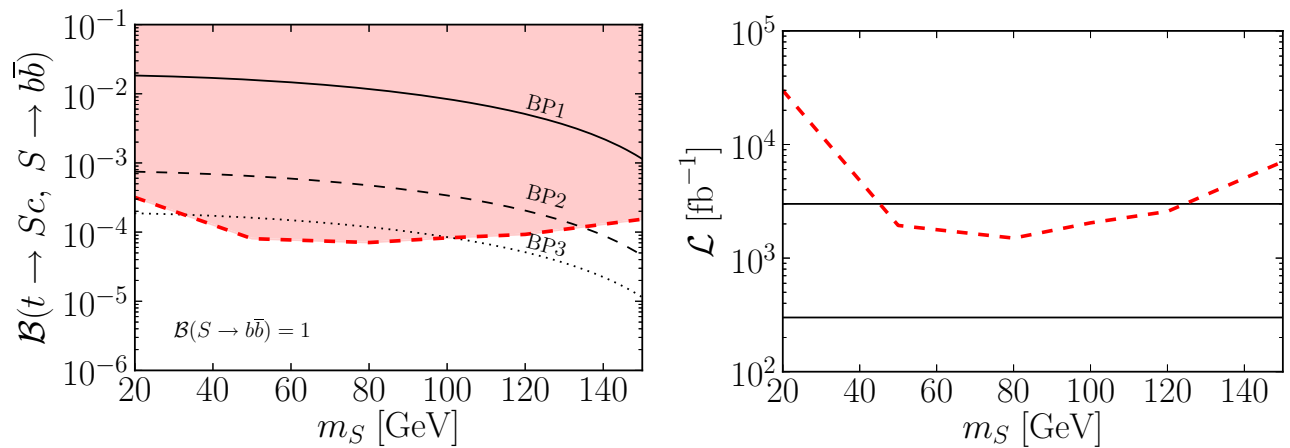

Fig. 2 (Left) Branching ratios that can be tested in the $b \bar{b}$ channel. Superimposed are the theoretical expectations in the three BPs. (Right) Luminosity required to test $\mathcal{B}(t \rightarrow S c, S \rightarrow b \bar{b})=10^{-4}$. Superimposed are $\mathcal{L}=300 \mathrm{fb}^{-1}$ and $3000 \mathrm{fb}^{-1}$ 

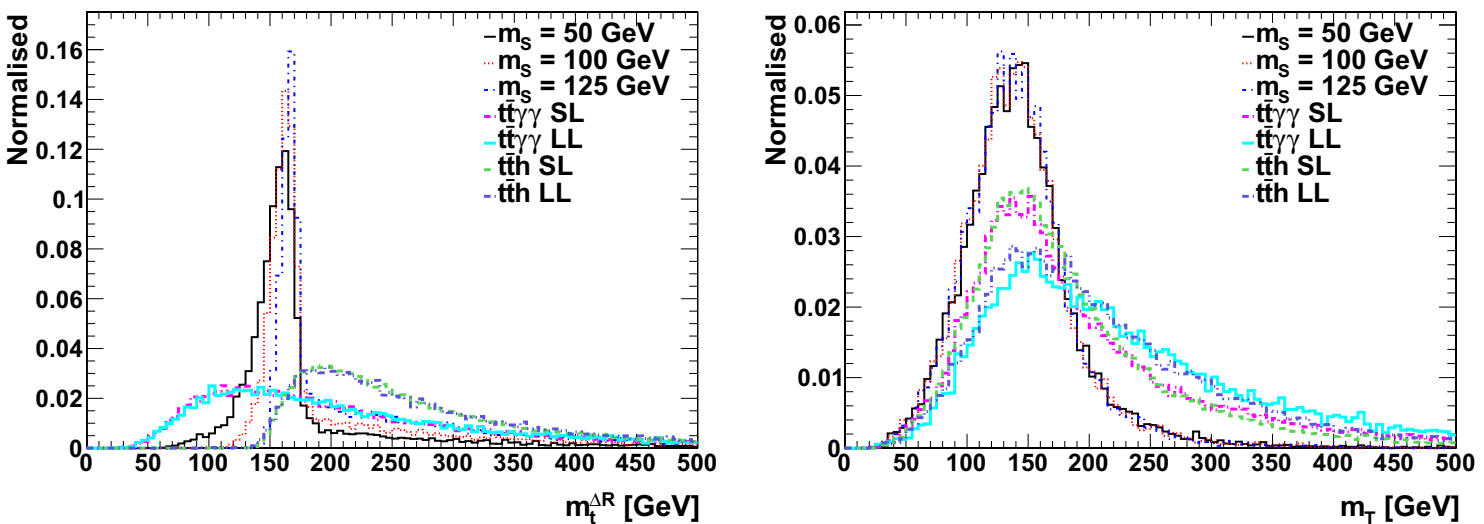

Fig. 3 (Left) The reconstructed top mass from the hardest two photons and the hardest jet. (Right) The transverse mass $m_{T}$

Table 5 Efficiency after each cut for the seven signal benchmark points

\begin{tabular}{|c|c|c|c|c|c|c|c|}
\hline Cuts & $20 \mathrm{GeV}$ & $50 \mathrm{GeV}$ & $80 \mathrm{GeV}$ & $100 \mathrm{GeV}$ & $120 \mathrm{GeV}$ & $125 \mathrm{GeV}$ & $150 \mathrm{GeV}$ \\
\hline Basic & 0.18 & 0.18 & 0.18 & 0.17 & 0.16 & 0.15 & 0.12 \\
\hline$\left|\eta_{(b, \ell, j, \gamma)}\right|<2.5$ & 0.91 & 0.91 & 0.91 & 0.90 & 0.89 & 0.88 & 0.83 \\
\hline$\Delta R($ all pairs $)>0.4$ & 0.62 & 0.91 & 0.91 & 0.90 & 0.88 & 0.88 & 0.87 \\
\hline$\left|m_{t}^{\text {reco }}-m_{t}\right|<50 \mathrm{GeV}$ & 0.81 & 0.78 & 0.77 & 0.74 & 0.65 & 0.61 & 0.33 \\
\hline$m_{T}<200 \mathrm{GeV}$ & 0.93 & 0.92 & 0.92 & 0.93 & 0.93 & 0.94 & 0.94 \\
\hline
\end{tabular}

Table 6 Efficiency after each cut for the four dominant backgrounds. SL (LL) denotes semi (di)-leptonic decays

\begin{tabular}{lllll}
\hline Cuts & $t \bar{t} \gamma \gamma(\mathrm{SL})$ & $t \bar{t} \gamma \gamma(\mathrm{LL})$ & $t \bar{t} h(\mathrm{SL})$ & $t \bar{t} h(\mathrm{LL})$ \\
\hline Basic & 0.18 & 0.12 & 0.26 & 0.16 \\
$\left|\eta_{(b, \ell, j, \gamma)}\right|<2.5$ & 0.94 & 0.92 & 0.94 & 0.89 \\
$\Delta R($ all pairs $)>0.4$ & 0.86 & 0.72 & 0.88 & 0.79 \\
$\left|m_{t}^{\text {reco }}-m_{t}\right|<50 \mathrm{GeV}$ & 0.37 & 0.36 & 0.38 & 0.37 \\
$m_{T}<200 \mathrm{GeV}$ & 0.65 & 0.50 & 0.71 & 0.60 \\
\hline
\end{tabular}

\section{LHC prospects for $t \rightarrow S c, S \rightarrow \gamma \gamma$}

We closely follow the previous section in terms of the analysis framework. Here we focus on the scenario in which the scalar decays to a pair of photons, yielding the final state with at least two jets, with one being $b$-tagged, one isolated lepton and two isolated photons. Similar to the leptons, we require the photons to have $p_{T}>10 \mathrm{GeV}$ and require them to lie within a pseudorapidity range of $|\eta|<2.5$. We demand the photons to be isolated with the exact same criteria as for the leptons as discussed in the section above. The $\Delta R>0.4$ cuts between pairs of all the selected objects are also used for this study. The dominant backgrounds for this channel are the semi-leptonic and di-leptonic $t \bar{t} h$ processes and the QCDQED production of $t \bar{t} \gamma \gamma$. The cross section of the former is scaled by a $K$-factor of 1.68 , what takes into account the NLO corrections to both the production and the $h$ decay [58]. For the second, we use a conservative $K$-factor of 2 . We also include the $W \gamma \gamma$ background matched up to two hard jets.
However, despite having a cross section of order $\mathcal{O}(0.1) \mathrm{pb}$, it becomes irrelevant after imposing all cuts. Consequently, we do not show explicit numbers for this process hereafter.

The selection level cuts up until the transverse mass are identical to the previous section. However, because of a much sharper diphoton mass resolution, we demand a very narrow window of $3 \mathrm{GeV}$ around the scalar mass. The shape of the reconstructed top mass distributions as well as $m_{T}$ in this case are shown in Fig. 3 after the basic cuts (which includes objects selected with the $p_{T}$ requirement as mentioned above along with the selection criteria of exactly one $b$-tagged jet, at least one additional jet, one isolated lepton and two or more isolated photons). The cutflows are listed in Tables 5, 6 and 7. The 95\% CL upper limit on the branching ratio $\mathcal{B}(t \rightarrow$ $S c, S \rightarrow \gamma \gamma)$ is shown in Fig. 4 along with the minimum integrated luminosity required to probe a branching ratio of $10^{-6}$. In this analysis we have added a new $m_{S}$ mass point of $125 \mathrm{GeV}$, where the $t \bar{t} h$ background is much larger. The dominance of this latter process is apparent in the figure. 
Table 7 Efficiencies $\left(\times 10^{4}\right)$ after the final cut, $\left|m_{\gamma \gamma}-m_{S}\right|<3 \mathrm{GeV}$, for each signal benchmark point and for the corresponding backgrounds. SL (LL) denotes semi (di)-leptonic decays

\begin{tabular}{llllll}
\hline$m_{S}(\mathrm{GeV})$ & Signal & $t \bar{t} \gamma \gamma(\mathrm{SL})$ & $t \bar{t} \gamma \gamma(\mathrm{LL})$ & $t \bar{t} h(\mathrm{SL})$ & $t \bar{t} h(\mathrm{LL})$ \\
\hline 20 & 760 & 13 & 5.5 & 0.15 & 0.20 \\
50 & 1100 & 27 & 9.9 & 0.40 & 0.25 \\
80 & 1000 & 19 & 6.8 & 0.45 & 0.35 \\
100 & 940 & 13 & 5.0 & 0.20 & 0.25 \\
120 & 740 & 6.4 & 3.5 & 0.25 & 0.35 \\
125 & 660 & 5.0 & 2.6 & 570 & 240 \\
150 & 280 & 2.3 & 1.1 & 0.00 & 0.00 \\
\hline
\end{tabular}
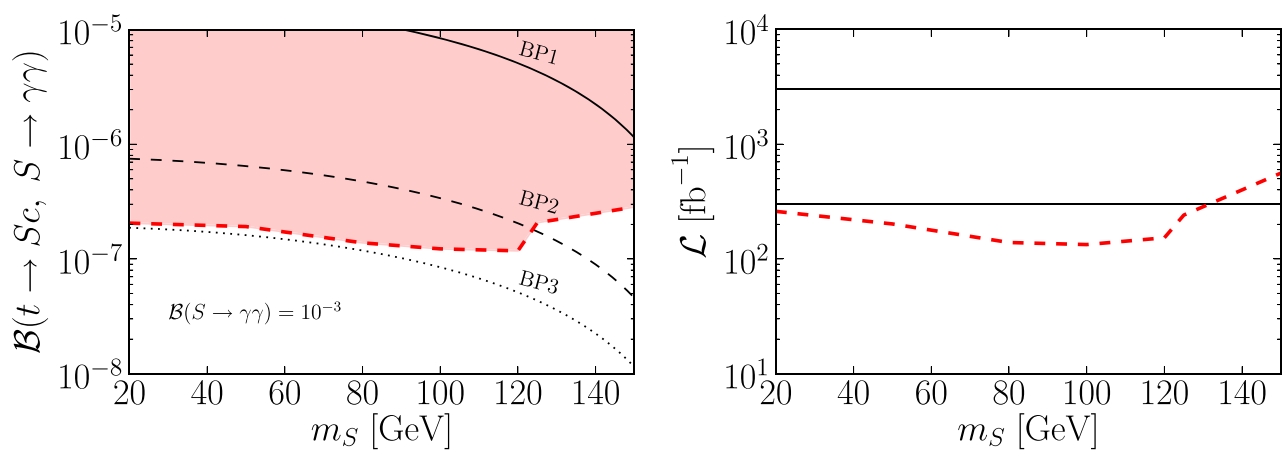

Fig. 4 (Left) Branching ratios that can be tested in the $\gamma \gamma$ channel. Superimposed are the theoretical expectations in the three BPs. (Right) Luminosity required to test $\mathcal{B}(t \rightarrow S t, S \rightarrow \gamma \gamma)=10^{-6}$. Superimposed are $\mathcal{L}=300 \mathrm{fb}^{-1}$ and $3000 \mathrm{fb}^{-1}$

\section{Conclusions}

Flavour-violating top decays into scalar singlets, $S$, mediated by new physics at a scale $f \gtrsim \mathcal{O}(\mathrm{TeV})$, dominate strongly over the ones involving the SM-like Higgs boson. From an effective-field theory point of view, the main reason for the dominance of the new scalar is due to the fact that the latter proceeds via effective operators of dimension six and is hence suppressed by $1 / f^{2}$, whereas the former is already present at dimension five (and hence suppressed only by $1 / f)$. Moreover, the singlet can be much lighter than the Higgs, the corresponding top decay being therefore kinematically enhanced. Since such scalar particles are predicted in several new physics models, we designed novel analyses dedicated for the upcoming runs of the LHC, to search for $t \rightarrow S c, S \rightarrow b \bar{b} / \gamma \gamma$ in events pertaining to top pair production. We restricted our study of $S$ masses varying between $20 \mathrm{GeV}<m_{S}<150 \mathrm{GeV}$.

In the $S \rightarrow b \bar{b}$ channel, the highest reach is obtained for $m_{S} \sim 80 \mathrm{GeV}$, for which we can probe $\mathcal{B}(t \rightarrow S c, S \rightarrow$ $b \bar{b})>10^{-4}$ at $95 \% \mathrm{CL}$ with an integrated luminosity of $\mathcal{L}=3 \mathrm{ab}^{-1}$. The reach is about a factor of 5 smaller for low masses. This is due to the fact that at low masses the two $b$-quarks ensuing from the scalar $S$, do not always form two resolved $b$-jets, and hence upon requiring three $b$-tagged jets, we incur a reduction in the efficiency of the signal. However, one might consider a fat jet in the framework of a boosted analysis to overcome this difficulty. On the other hand, for large masses, the invariant mass of the two $b$-tagged jets closest in $\Delta R$ separation, do not always peak at $m_{S}$.

In the $\gamma \gamma$ channel, the sensitivity of the signal is considerably less dependent on $m_{S}$, given an excellent resolution of the di-photon mass spectrum. We find that a branching ratio, $\mathcal{B}(t \rightarrow S c, S \rightarrow \gamma \gamma)>10^{-7}$ can be tested at the $95 \% \mathrm{CL}$ with the same integrated luminosity. We note that, if $\mathcal{B}(S \rightarrow \gamma \gamma)$ is as large as $\sim 1 \%$, then we can indirectly probe new physics scales as large as $\sim 50 \mathrm{TeV}$. Furthermore, we note that the bound obtained in this channel for $m_{S} \sim m_{h}$ agrees well with the results obtained for $t \rightarrow h c$ listed in previous works [22] (which utilise significantly different search strategies and statistical approaches). Reference [22] also showed that the increase in sensitivity at a $100 \mathrm{TeV}$ collider can be roughly estimated by scaling the signal and background cross-sections and the luminosity. In this particular channel, the dominant background is $t \bar{t} \gamma \gamma$ for masses of the singlet, $m_{S}$, well separated from $m_{h}$. It turns out that the increase in cross section in this background at $\sqrt{s}=27 \mathrm{TeV}(100 \mathrm{TeV})$ with respect to that at $\sqrt{s}=14 \mathrm{TeV}$ is similar to that in the signal, and of order $\sim 4(\sim 40)$. Thus, assuming an integrated luminosity of $10 \mathrm{ab}^{-1}$, we expect an increase in significance of order $4 / \sqrt{4} \times \sqrt{10 / 3} \sim 3.7$ $(40 / \sqrt{40} \times \sqrt{10 / 3} \sim 11.5)$. This implies that one can expect up to an order of magnitude improvement in the bound on $\mathcal{B}(t \rightarrow S c, S \rightarrow \gamma \gamma)$. Similar results will hold for the $b \bar{b}$ channel. 
Acknowledgements We acknowledge Shilpi Jain, Maria Ramos, Jose Santiago and Jakub Scholtz for useful discussions. MC is supported by the Royal Society under the Newton International Fellowship programme. SB is supported by a Durham Junior Research Fellowship COFUNDed between Durham University and the European Union under Grant Agreement Number 609412.

Open Access This article is distributed under the terms of the Creative Commons Attribution 4.0 International License (http://creativecomm ons.org/licenses/by/4.0/), which permits unrestricted use, distribution, and reproduction in any medium, provided you give appropriate credit to the original author(s) and the source, provide a link to the Creative Commons license, and indicate if changes were made.

Funded by SCOAP ${ }^{3}$.

\section{References}

1. T.M.P. Tait, C.P. Yuan, Anomalous t-c-g coupling: The connection between single top production and top decay. Phys. Rev. D 55, 7300-7301 (1997). arXiv:hep-ph/9611244

2. J. Guasch, W. Hollik, S. Penaranda, J. Sola, Single top-quark production by direct supersymmetric flavor-changing neutral-current interactions at the LHC. Nucl. Phys. Proc. Suppl. 157, 152-156 (2006). arXiv:hep-ph/0601218

3. T. Plehn, M. Rauch, M. Spannowsky, Understanding single tops using Jets. Phys. Rev. D 80, 114027 (2009). arXiv:0906.1803

4. M. Blanke, G.F. Giudice, P. Paradisi, G. Perez, J. Zupan, Flavoured naturalness. JHEP 06, 022 (2013). arXiv: 1302.7232

5. C. Degrande, F. Maltoni, J. Wang, C. Zhang, Automatic computations at next-to-leading order in QCD for top-quark flavorchanging neutral processes. Phys. Rev. D 91, 034024 (2015). arXiv: 1412.5594

6. R. Goldouzian, Search for top quark flavor changing neutral currents in same-sign top quark production. Phys. Rev. D 91, 014022 (2015). arXiv:1408.0493

7. M. Backović, A. Mariotti, M. Spannowsky, Signs of tops from highly mixed stops. JHEP 06, 122 (2015). arXiv:1504.00927

8. K. Agashe, R. Contino, Composite Higgs-mediated FCNC. Phys. Rev. D 80, 075016 (2009). arXiv:0906.1542

9. B. Mele, S. Petrarca, A. Soddu, A new evaluation of the $t \rightarrow c H$ decay width in the standard model. Phys. Lett. B 435, 401-406 (1998). arXiv:hep-ph/9805498

10. A. Greljo, J.F. Kamenik, J. Kopp, Disentangling flavor violation in the top-higgs sector at the LHC. JHEP 07, 046 (2014). arXiv: 1404.1278

11. A. Azatov, G. Panico, G. Perez, Y. Soreq, On the flavor structure of natural composite Higgs models \& top flavor violation. JHEP 12, 082 (2014). arXiv: 1408.4525

12. CMS collaboration, V. Khachatryan et al., Search for anomalous single top quark production in association with a photon in pp collisions at $\sqrt{s}=8 \mathrm{TeV}$. JHEP 04, 035 (2016). arXiv:1511.03951

13. F.J. Botella, G.C. Branco, M. Nebot, M.N. Rebelo, Flavour changing Higgs couplings in a class of two Higgs doublet models. Eur. Phys. J. C 76, 161 (2016). arXiv: 1508.05101

14. D. Bardhan, G. Bhattacharyya, D. Ghosh, M. Patra, S. Raychaudhuri, Detailed analysis of flavor-changing decays of top quarks as a probe of new physics at the LHC. Phys. Rev. D 94, 015026 (2016). arXiv: 1601.04165

15. M. Badziak, K. Harigaya, Asymptotically free natural SUSY twin Higgs. Phys. Rev. Lett. 120, 211803 (2018). arXiv: 1711.11040

16. CMS collaboration, V. Khachatryan et al., Search for anomalous Wtb couplings and flavour-changing neutral currents in t-channel single top quark production in pp collisions at $\sqrt{s}=7$ and $8 \mathrm{TeV}$. JHEP 02, 028 (2017). arXiv: 1610.03545
17. E. Gabrielli, B. Mele, M. Raidal, E. Venturini, FCNC decays of standard model fermions into a dark photon. Phys. Rev. D 94, 115013 (2016). arXiv: 1607.05928

18. CMS Collaboration, Search for flavour changing neutral currents in top quark production and decays with three-lepton final state using the data collected at sqrt(s) $=13 \mathrm{TeV}$, Tech. Rep. CMS-PASTOP-17-017, CERN, Geneva (2017)

19. ATLAS collaboration, M. Aaboud et al., Search for flavourchanging neutral current top-quark decays $t \rightarrow q Z$ in protonproton collisions at $\sqrt{s}=13 \mathrm{TeV}$ with the ATLAS detector. arXiv: 1803.09923

20. ATLAS collaboration, M. Aaboud et al., Search for top quark decays $t \rightarrow q H$, with $H \rightarrow \gamma \gamma$, in $\sqrt{s}=13 \mathrm{TeV} p p$ collisions using the ATLAS detector. JHEP 10, 129 (2017). arXiv: 1707.01404

21. ATLAS collaboration, M. Aaboud et al., Search for flavor-changing neutral currents in top quark decays $t \rightarrow H c$ and $t \rightarrow H u$ in multilepton final states in proton-proton collisions at $\sqrt{s}=13$ TeV with the ATLAS detector. arXiv: 1805.03483

22. A. Papaefstathiou, G. Tetlalmatzi-Xolocotzi, Rare top quark decays at a $100 \mathrm{TeV}$ protonproton collider: $t \rightarrow b W Z$ and $t \rightarrow h c$. Eur. Phys. J. C 78, 214 (2018). arXiv: 1712.06332

23. N. Craig, J.A. Evans, R. Gray, M. Park, S. Somalwar, S. Thomas, Searching for $t \rightarrow c h$ with multi-Leptons. Phys. Rev. D 86, 075002 (2012). arXiv:1207.6794

24. R. Harnik, J. Kopp, J. Zupan, Flavor violating Higgs decays. JHEP 03, 026 (2013). arXiv: 1209.1397

25. ATLAS, CMS collaboration, G. Aad et al., Measurements of the Higgs boson production and decay rates and constraints on its couplings from a combined ATLAS and CMS analysis of the LHC pp collision data at $\sqrt{s}=7$ and $8 \mathrm{TeV}$. JHEP 08, 045 (2016). arXiv: 1606.02266

26. S. Banerjee, M. Mitra, M. Spannowsky, Searching for a heavy Higgs boson in a Higgs-portal B-L Model. Phys. Rev. D 92, 055013 (2015). arXiv:1506.06415

27. DELPHI, OPAL, ALEPH, LEP Electroweak Working Group, L3 collaboration, J. Alcaraz et al., A combination of preliminary electroweak measurements and constraints on the standard model. arXiv:hep-ex/0612034

28. CDF collaboration, T. Aaltonen et al., Precise measurement of the $W$-boson mass with the CDF II detector. Phys. Rev. Lett. 108, 151803 (2012). arXiv: 1203.0275

29. D0 collaboration, V.M. Abazov et al., Measurement of the $W$ boson mass with the D0 detector. Phys. Rev. D 89, 012005 (2014). arXiv: 1310.8628

30. T. Robens, T. Stefaniak, Status of the Higgs singlet extension of the standard model after LHC Run 1. Eur. Phys. J. C 75, 104 (2015). arXiv: 1501.02234

31. D. Lpez-Val, T. Robens, $\mathrm{r}$ and the W-boson mass in the singlet extension of the standard model. Phys. Rev. D 90, 114018 (2014). arXiv: 1406.1043

32. M.E. Peskin, Comparison of LHC and ILC capabilities for Higgs Boson coupling measurements. arXiv: 1207.2516

33. N. Craig, H.K. Lou, M. McCullough, A. Thalapillil, The Higgs portal above threshold. JHEP 02, 127 (2016). arXiv:1412.0258

34. U. Ellwanger, C. Hugonie, A.M. Teixeira, The next-to-minimal supersymmetric standard model. Phys. Rept. 496, 1-77 (2010). arXiv:0910.1785

35. S. Dimopoulos, J. Preskill, Massless composites with massive constituents. Nucl. Phys. B 199, 206-222 (1982)

36. D.B. Kaplan, H. Georgi, SU(2) x U(1) breaking by vacuum misalignment. Phys. Lett. B 136, 183-186 (1984)

37. D.B. Kaplan, H. Georgi, S. Dimopoulos, Composite Higgs scalars. Phys. Lett. B 136, 187-190 (1984)

38. G. Panico, A. Wulzer, The composite Nambu-Goldstone Higgs. Lect. Notes Phys. 913, 1-316 (2016). arXiv:1506.01961 
39. C. Zhang, F. Maltoni, Top-quark decay into Higgs boson and a light quark at next-to-leading order in QCD. Phys. Rev. D 88, 054005 (2013). arXiv:1305.7386

40. UTfit collaboration, M. Bona et al., Model-independent constraints on $\Delta F=2$ operators and the scale of new physics. JHEP 03, 049 (2008). arXiv:0707.0636

41. Top Quark Working Group collaboration, K. Agashe et al., Working Group Report: Top Quark, in Proceedings, 2013 Community Summer Study on the Future of U.S. Particle Physics: Snowmass on the Mississippi (CSS2013): Minneapolis, MN, USA, July 29-August 6, 2013 (2013). arXiv:1311.2028

42. CMS collaboration, Search for the flavor-changing interactions of the top quark with the Higgs boson in $H \rightarrow b \bar{b}$ channel at $\sqrt{s}=$ $13 \mathrm{TeV}$, CMS-PAS-TOP-17-003 (2017)

43. Particle Data Group collaboration, C. Patrignani et al., Review of particle physics. Chin. Phys. C 40, 100001 (2016)

44. B. Gripaios, A. Pomarol, F. Riva, J. Serra, Beyond the minimal composite Higgs model. JHEP 04, 070 (2009). arXiv:0902.1483

45. G. Ferretti, D. Karateev, Fermionic UV completions of composite Higgs models. JHEP 03, 077 (2014). arXiv:1312.5330

46. M. Chala, R. Grber, M. Spannowsky, Searches for vector-like quarks at future colliders and implications for composite Higgs models with dark matter. JHEP 03, 040 (2018). arXiv:1801.06537

47. G.C. Branco, D. Emmanuel-Costa, R. Gonzalez, Yukawa structure with maximal predictability. Phys. Lett. B 483, 87-93 (2000). arXiv:hep-ph/9905290

48. R.G. Roberts, A. Romanino, G.G. Ross, L. Velasco-Sevilla, Precision test of a fermion mass texture. Nucl. Phys. B 615, 358-384 (2001). arXiv:hep-ph/0104088
49. CMS collaboration, V. Khachatryan et al., Search for Higgs boson off-shell production in proton-proton collisions at 7 and $8 \mathrm{TeV}$ and derivation of constraints on its total decay width. JHEP 09, 051 (2016). arXiv:1605.02329

50. J.E. Kim, H.P. Nilles, The mu problem and the strong CP problem. Phys. Lett. B 138, 150-154 (1984)

51. J. Alwall, R. Frederix, S. Frixione, V. Hirschi, F. Maltoni, O. Mattelaer, The automated computation of tree-level and next-toleading order differential cross sections, and their matching to parton shower simulations. JHEP 07, 079 (2014). arXiv:1405.0301

52. M.L. Mangano, M. Moretti, F. Piccinini, M. Treccani, Matching matrix elements and shower evolution for top-quark production in hadronic collisions. JHEP 01, 013 (2007). arXiv:hep-ph/0611129

53. LHC Physics: NNLO+NNLL top-quark-pair cross sections. https://twiki.cern.ch/twiki/bin/view/LHCPhysics/TtbarNNLO Accessed 22 Aug 2018

54. R.D. Ball, Parton distributions with LHC data. Nucl. Phys. B 867, 244-289 (2013). arXiv:1207.1303

55. T. Sjöstrand, S. Ask, J.R. Christiansen, R. Corke, N. Desai, P. Ilten, An Introduction to PYTHIA 8.2. Comput. Phys. Commun. 191, 159-177 (2015). arXiv:1410.3012

56. M. Cacciari, G.P. Salam, G. Soyez, The Anti-k(t) jet clustering algorithm. JHEP 04, 063 (2008). arXiv:0802.1189

57. M. Cacciari, G.P. Salam, G. Soyez, FastJet user manual. Eur. Phys. J. C 72, 1896 (2012). arXiv:1111.6097

58. LHCPhysics (2014). http://twiki.cern.ch/twiki/bin/view/ lhcphysics/cernyellowreportpageat1314tev2014 